\title{
Une pirogue chez les Iatmul du fleuve Sépik (Papouasie Nouvelle-Guinée)
}

A iatmul pirogue of the Sepik river (Papua-New-Guinea)

Una piragua iatmul del río Sepik (Papuasia Nueva Guinea)

\section{Christian Coiffier}

\section{(2) OpenEdition}

Journals

Édition électronique

URL : https://journals.openedition.org/tc/291

DOI : 10.4000/tc. 291

ISSN : 1952-420X

Éditeur

Éditions de l'EHESS

Édition imprimée

Date de publication : 1 janvier 2001

Pagination : 233-254

ISSN : 0248-6016

Référence électronique

Christian Coiffier, «Une pirogue chez les latmul du fleuve Sépik (Papouasie Nouvelle-Guinée) », Techniques \& Culture [En ligne], 35-36 | 2001, mis en ligne le 07 septembre 2012, consulté le 29

septembre 2022. URL : http://journals.openedition.org/tc/291 ; DOI : https://doi.org/10.4000/tc.291

Ce document a été généré automatiquement le 29 septembre 2022.

Tous droits réservés 


\title{
Une pirogue chez les Iatmul du fleuve Sépik (Papouasie Nouvelle- Guinée)
}

\author{
A iatmul pirogue of the Sepik river (Papua-New-Guinea) \\ Una piragua iatmul del río Sepik (Papuasia Nueva Guinea)
}

\section{Christian Coiffier}

1 Les Iatmul ${ }^{2}$ forment une société d'environ dix milles horticulteurs-pêcheurs, installés sur un vaste territoire marécageux baigné par le fleuve Sépik (situé au nord-ouest de l'État de Papouasie Nouvelle-Guinée). Ils vivent encore partiellement dans une économie de subsistance fondée sur la pêche. Ce sont essentiellement les femmes qui pratiquent cette activité pour nourrir la famille. Elles échangent le surplus de poisson contre de la farine de sagou produite par les femmes des peuples voisins vivant dans les marécages situés en arrière du lit principal du fleuve, les peuples Sawos au nord, et ceux de la région des lacs: Chambri, Blackwater et Kovenmas au sud (fig. 1). 


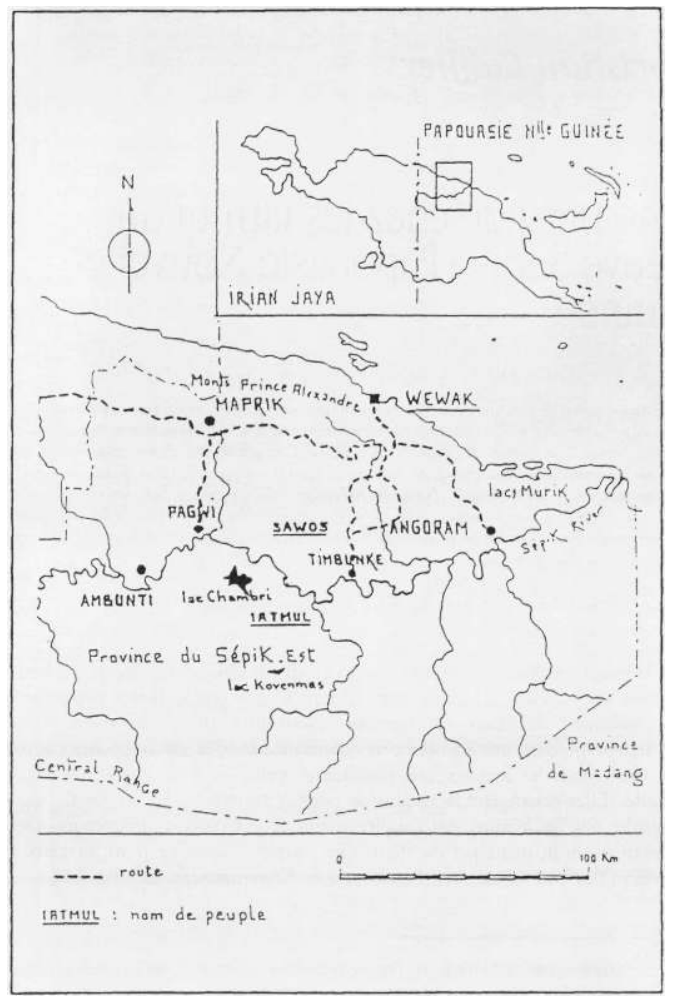

Figure 1. Carte de la région iatmul avec les principaux villages et routes

2 Les villages et les jardins iatmul sont édifiés sur des plates-formes limoneuses constituant les berges temporaires du fleuve Sépik. Chaque année, lors de la crue, du mois de décembre au mois de mars, le niveau de l'eau monte de plusieurs mètres, submergeant les berges et une grande partie de la vallée. Les maisons sur pilotis se retrouvent ainsi entourées d'eau. Lors des crues exceptionnelles, tous les dix ans environ, l'eau peut atteindre la base des toitures; de nombreux édifices sont alors détruits et emportés par le puissant courant. Il n'est donc pas étonnant que les Iatmul perçoivent leur territoire comme un conglomérat d'îles flottantes (Coiffier 1995b).

Chacun des villages se trouve divisé en deux moitiés totémiques patri-linéaires; d'un côté les Nyamenemba, associés à la terre et à la nuit, de l'autre les Nyawinemba, associés au soleil et au jour; mais cette division s'est souvent altérée avec la christianisation. Chaque village, équivalent symbolique d'un crocodile, possède au moins une grande maison cérémonielle qui est le centre rituel de la communauté. Située au milieu d'une longue place, réservée aux seuls hommes initiés, elle était autrefois perçue, également, comme une île flottante sous laquelle vivaient les hommes-crocodiles (Bateson 1971 : 289). Jusqu'à la dernière guerre mondiale, ces divers groupes étaient en conflit permanent. La « chasse aux têtes " était au centre de leur vie sociale et faisait partie d'un ensemble complexe de rituels reposant sur le principe dualiste de la division du macrocosme comme du microcosme. Les têtes ainsi appropriées étaient censées assurer la prospérité des communautés (Coiffier et Guerreiro 1999).

Indispensable outil de production et de déplacement (photo 1), la pirogue permet aux femmes de se rendre sur leurs lieux de pêche pour traîner leur haveneau, placer des lignes de fond dans les hautes herbes des berges et relever les nasses placées dans les petits chenaux. Elle assure en outre le transport des personnes et des marchandises ${ }^{3}$ 
entre les différents villages (photo 2). C'est aussi une des représentations du crocodile ancestral qui protège ses utilisateurs. Comme telle, elle est souvent employée lors des rituels les plus importants.

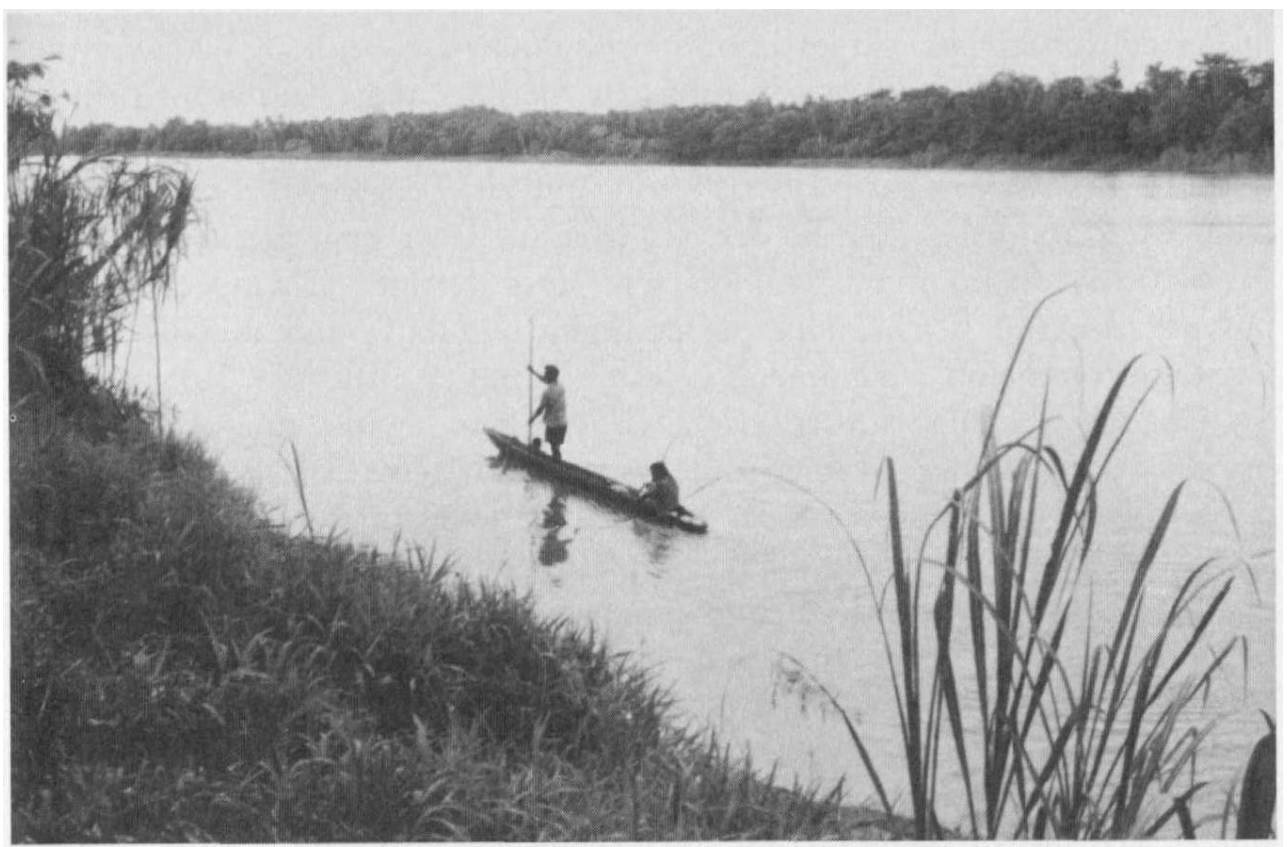

Photo1. Famille remontant le cours du fleuve à Abusatngeï (1988)

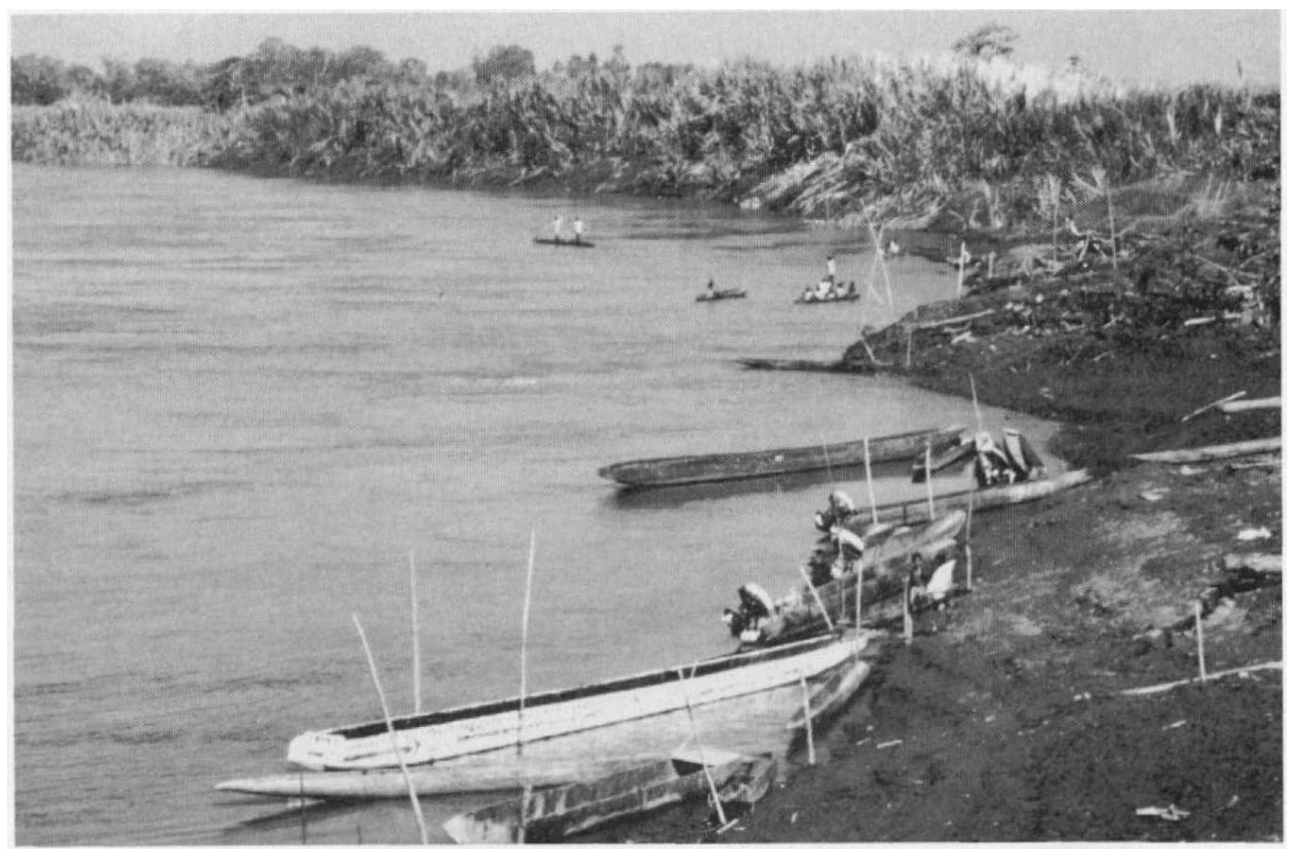

Photo 2. Débarcadère des grandes pirogues près du village de Pagwi (1988)

\section{La fabrication des pirogues}

Le tableau ci-dessous énumère les différents types de pirogue ${ }^{4}$ que réalisent les Iatmul ${ }^{5}$. 


\begin{tabular}{|l|l|l|l|}
\hline Nom & Longueur & Contenance & Fonction \\
\hline makwala & 3 à $4 \mathrm{~m}$ & 1 ou 2 enfants & pêche et jeux \\
\hline kunguwala & 5 à $8 \mathrm{~m}$ & 2 à 3 femmes & pêche \\
\hline wengawala & 10 à $13 \mathrm{~m}$ & 5 à 7 femmes & pêche/transport \\
\hline nemawala & 15 à $20 \mathrm{~m}$ & 5 à 15 hommes & transport \\
\hline yampuenguewala & près de $20 \mathrm{~m}$ & 15 à 20 hommes et femmes & commerce \\
\hline kambukwala & plus de $20 \mathrm{~m}$ & 15 à 20 hommes & guerre \\
\hline
\end{tabular}

Ce sont les hommes qui ont en charge la fabrication de ces pirogues, selon les besoins et en toute saison. En principe, la période de crue est propice à cette activité, car les hommes ne peuvent travailler dans les jardins inondés ; ils profitent donc de la montée des eaux pour haler les troncs par flottage sur les tertres émergés. Ils travaillent ainsi à la réalisation de leur nouvelle pirogue près de leur demeure.

De très nombreuses essences d'arbres peuvent servir à la fabrication, mais certaines d'entre elles sont plus particulièrement recherchées pour leurs qualités techniques (voir annexe). Certains bois, très denses, comme le miamba (Vitex cofassus) et le kwarap (Intsia bijunga) sont très appréciés. En général, la proue est réalisée dans la partie haute du tronc alors que la poupe est taillée dans la partie basse, plus dure. Toutefois, les bois tendres de certains arbres comme le kam (Artocarpus altilis) servent à la fabrication des petites pirogues d'enfants et de femmes.

8 La conception anthropomorphique des arbres et leur étroite relation avec le monde ancestral ritualisent leur traitement. L'abattage d'un arbre de 30 à 50 mètres de haut sur plus d'un mètre de diamètre à la base pose autant de problèmes techniques qu'éthiques. Une telle entreprise ne peut être menée par n'importe qui et n'importe comment, car un travail mal conduit peut entraîner mort d'homme de par la vengeance de l'esprit de l'arbre.

Les gros arbres sont souvent abattus avant leur période de floraison, c'est-à-dire, pour la plupart d'entre eux, avant la période de crue, en octobre-novembre. Lorsque l'arbre a été désigné et que son propriétaire est d'accord, le rituel est simple. Une date est choisie pour l'abattage. Il est fort probable que ce jour soit fixé par les big-men en fonction des phases de la lune, mais je n'en ai pas confirmation. Dans la matinée du jour suivant, les hommes se rendent auprès de l'arbre. Les opérations se déroulent sous la direction d'un ancien qui connaît parfaitement les rites à accomplir. Plusieurs hommes s'attaquent simultanément à la base du tronc (à plus d'un mètre de hauteur). Comme de nombreux arbres présentent des racines contreforts, celles-ci doivent être sectionnées avant de s'attaquer au tronc proprement dit. Il est donc parfois indispensable de construire une petite plate-forme (djamb), sur laquelle les hommes doivent grimper pour être à la bonne hauteur de coupe. En général, un arbre est entouré d'autres arbres, parfois porteurs de fruits ou de graines (manguiers, cocotiers, aréquiers, etc.). Il faut donc éviter que l'arbre coupé ne s'abatte sur ses voisins. Ainsi, lorsque le tronc est à moitié sectionné, les bûcherons s'adressent à l'arbre en ces termes : " Toi, arbre, tu ne dois pas t'abattre sur les autres arbres. Tu dois tomber du côté où il n'y a pas d'obstacles ». Les bûcherons s'attaquent alors au dos de l'arbre et le sectionnent jusqu'à ce qu'il bascule dans la bonne direction. Si un tronc, en tombant, détériore les arbres voisins, le propriétaire de ces derniers devra recevoir une compensation pour les dommages occasionnés. 
10 Un homme initié peut se permettre de choisir un arbre dans la "forêt tabou " (malingeko) du village. Mais avant de le couper, il doit obtenir l'accord du propriétaire, le père de l'arbre. Certains propriétaires demandent de l'argent, d'autres se contentent d'un modeste cadeau en nourriture, selon les relations qu'ils entretiennent avec le demandeur. Mais, dans tous les cas, un rituel doit être pratiqué avant l'abattage. Les hommes chargés de cette besogne se réunissent la veille pour préparer l'entreprise. Ils sont soumis à des interdits, comme par exemple la nécessité de s'abstenir de relations sexuelles avec leurs épouses. Des hommes viennent alors souffler dans de longues flûtes en bambou à quatre reprises, tandis que celui qui désire couper l'arbre frappe le sol quatre fois avec un balai en feuilles de cocotier. Au même moment, dans la maison cérémonielle, d'autres hommes frappent sur un grand tambour à fente qui évoque la voix de l'ancêtre crocodile, wagan (Coiffier 2000 : 197). Il existe en effet une relation analogique entre les grands tambours et les troncs d'arbres avec lesquels ils sont fabriqués (Bateson 1971).

11 Les arbres miamba (Vitex cofassus), appelés garamut en pidgin, sont considérés comme la chair de certains esprits ancestraux ${ }^{7}$.

12 Avant l'arrivée des Européens, deux types d'outils permettaient de sectionner un arbre : la hache (mimbakalekula) et l'herminette (kalekula), constituées toutes deux d'une lame de pierre polie (kula) emmanchée sur un morceau de bois à l'aide d'une ligature de rotin. Mais depuis près d'un siècle, les lames métalliques ont remplacé les lames lithiques. Ces dernières ont néanmoins subsisté jusqu'à nos jours dans certains villages sawos.

13 Lorsque l'arbre est à terre, on coupe la cime de l'arbre (mao mi), puis les branches ${ }^{8}$ (sande mi). Les hommes qui effectuent cette tâche s'excusent auprès de l'esprit de l'arbre pour lui avoir ôté la tête et les bras. Ce rite est important dans une société où la décapitation s'avérait primordiale à l'époque -aujourd'hui révolue- de la chasse aux têtes. La présence dans les branchages de nids d'oiseaux ou d'insectes (abeilles, fourmis, termites...) et de certaines plantes épiphytes sont autant de signes qui peuvent être interprétés de façon divinatoire. Un tronc peut être ensuite sectionné en plusieurs morceaux qui serviront à fabriquer des objets divers (une pirogue et une sculpture, par exemple). Le tronc est débarrassé de son écorce (tshimbe mi), puis les hommes laissent les morceaux sur place et s'en retournent au village afin de se réunir une fois encore pour prononcer de nouvelles paroles d'apaisement envers «l'esprit» de l'arbre. Les troncs destinés à la construction d'une pirogue sont souvent dégrossis sur place, ce qui les allège de façon substantielle.

14 Il faut ensuite transporter les troncs; deux options sont possibles : le flottage ou le roulage. Lorsque les eaux de la crue envahissent la plaine, c'est l'époque favorable pour le transport des troncs d'arbre. Dans le cas de grumes de densité moyenne, celles-ci sont attachées directement à l'aide d'un rotin derrière une pirogue qui les tire ainsi jusqu'au lieu de destination. Cette solution a l'avantage de ne réclamer que peu de main-d'œuvre. Les grumes de forte densité qui ne peuvent flotter, comme le miamba, sont ligaturées à des troncs de mam (Althoffia pleiostigma) ou de mbanduan (Endospermum moluccanum) de très faible densité, qui permettent à l'ensemble de se maintenir à la surface de l'eau.

15 Le roulage impose un grand nombre de participants. De petits rondins (turu) sont disposés perpendiculairement (environ tous les mètres) le long d'un chemin forestier, qui constitue une sorte de «chemin de schlitte » (fig. 2). L'une des extrémités du tronc 
est émincée et trouée de façon à pouvoir y passer un rotin (mbal kuvu) qui est solidement noué. Cette technique est plus courante chez les Sawos que chez les Iatmul, qui se contentent souvent de ligaturer directement le rotin autour du tronc. Ensuite, selon la grosseur de la grume, une ou plusieurs dizaines de jeunes hommes et femmes se répartissent sur toute la longueur du rotin, de chaque côté et alternativement. L'opération se déroule sous la direction d'un ancien qui coordonne et guide l'avancée de la grume à l'aide d'un épieu. Au signal donné, chacun agrippe le rotin et tire de toutes ses forces. Ce travail se fait dans la joie et s'accompagne souvent de chants. La grume glisse très rapidement sur les rondins qui roulent sous la charge. Les efforts ne sont jamais soutenus très longtemps (quelques minutes au plus) et sont entrecoupés de pauses qui permettent aux travailleurs de reprendre des forces en grignotant quelques fruits de la forêt cueillis en chemin. En général, les jeunes participant à cette opération sont rémunérés en nourriture. Le propriétaire de la future pirogue se doit d'offrir de la nourriture (porc, chien, poulet) à ses neveux utérins venus l'aider9.

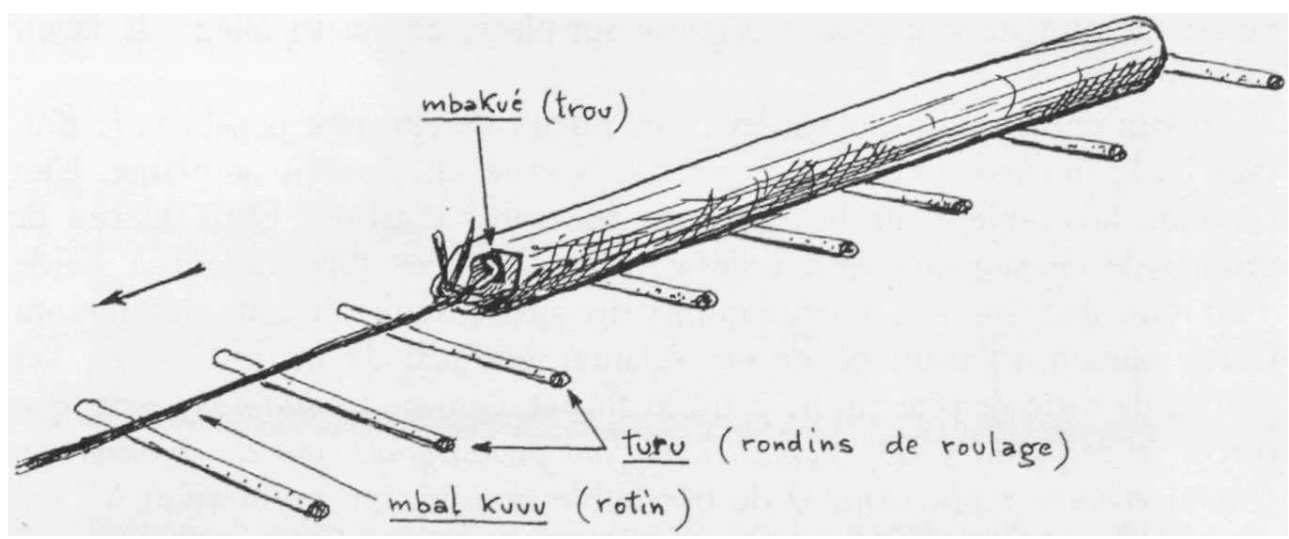

Figure 2. Technique de halage d'un tronc d'arbre

Les deux types de transport peuvent naturellement être combinés: roulage dans la forêt et flottage sut le fleuve jusqu'au village. Dans les mythes, la route de migration des arbres ancêtres est souvent celle d'un affluent du fleuve Sépik. Chaque clan iatmul revendique un certain nombre d'espèces d'arbres comme ancêtres de leurs différents lignages ${ }^{10}$.

La réalisation d'une grande pirogue nécessite plusieurs mètres cubes de bois ${ }^{11}$ et un bon mois de labeur ${ }^{12}$. La longueur d'une pirogue observée dans le village de Timbunke atteignait $17,50 \mathrm{~m}$ pour $1,04 \mathrm{~m}$ de large et $0,70 \mathrm{~m}$ de profondeur ; l'épaisseur des platsbords était de 6 à $8 \mathrm{~cm}$. Une autre pirogue, du village de Lukluk, comptait $17,15 \mathrm{~m}$ de long sur 1,15 $\mathrm{m}$ de large et $0,70 \mathrm{~m}$ de profondeur, avec une épaisseur de $4 \mathrm{~cm}$ pour les plats-bords (Coiffier 1994). Il est vraisemblable que certaines pirogues de guerre pouvaient atteindre plus de 20 mètres de long.

De grandes pirogues seulement ébauchées sont parfois achetées aux peuples Sawos vivant dans les collines et marécages au-delà des berges du fleuve. Ces derniers disposent en effet, sur leur territoire, d'une plus grande variété d'arbres. Autrefois, le troc se faisait contre des produits du fleuve, poissons et crustacés ; aujourd'hui, il se fait contre de l'argent. Les troncs sont abattus, dégrossis et évidés sommairement, puis halés par roulement, sur les chemins forestiers menant au fleuve (Coiffier 1994 : 947). Les Iatmul acquéreurs en assurent la finition et notamment la sculpture de proue. 


\section{Exemple de fabrication d'une petite pirogue}

19 Tambemali ${ }^{13}$ s'est procuré à Yentchen un morceau de tronc de wane (Octomeles sumatrana) de 6, $25 \mathrm{~m}$ de longueur sur $0,40 \mathrm{~m}$ de diamètre. Le tronc est resté longtemps dans l'eau du fleuve et il dégage une forte odeur de moisi. Après l'avoir traîné derrière sa pirogue jusqu'au hameau d'Abusatngeï, Tambemali se fait aider par ses enfants et petits-enfants pour le hisser sur la rive, en face de sa demeure.

20 Tambemali se met au travail à 16 heures 30 ; il observe attentivement le tronc, le retourne plusieurs fois avant de choisir le meilleur côté pour commencer. La proue (ndamawala) sera taillée du côté de la tête de l'arbre (kuasa mi), alors que la poupe (kwanguwala), plus large, le sera dans la partie proche du pied (angwa mi) ${ }^{14}$. Selon Tambemali, cette partie est plus dure et plus solide; elle est donc plus à même de résister à l'usure et aux chocs que subissent les poupes. J'ai pu constater les zones d'usure du dessous des poupes ${ }^{15}$ en observant les pirogues abandonnées.

21 Des cales (kungumi) sont placées de chaque côté du tronc pour l'empêcher de rouler lors de la découpe, puis ce dernier est attaqué à la hache et à l'herminette afin d'ébaucher la forme de la poupe. Le diamètre du tronc est réduit à une dimension de $35 \mathrm{~cm}$. Les copeaux (djingunu) obtenus sont jetés au fur et à mesure dans un petit feu. L'abondante fumée qui s'en dégage a l'avantage de faire fuir les moustiques, très nombreux dans cette région. Mais ces copeaux ne doivent surtout pas être brûlés dans un foyer domestique utilisé pour la cuisson de la nourriture ${ }^{16}$.

22 À 17 heures, la forme générale de l'arrière de la pirogue est déjà bien esquissée. Tambemali constitue ainsi une surface plane (sitia) sur la moitié du tronc en prenant bien soin de ménager les emplacements des accroches (mutmut). Il s'attaque ensuite au creusement du ventre (yale ble kula). La technique la plus courante consiste à tailler, sur toute la longueur du tronc, une longue excavation rectangulaire (pavu) indiquant grossièrement la forme de l'intérieur de la pirogue, puis à creuser des encoches de $10 \mathrm{~cm}$ de large (sungu), séparées par des cloisons (lavule djingu) d'environ $20 \mathrm{~cm}$ d'épaisseur (fig. 3). Quelques coups de hache et d'herminette suffisent ensuite à faire sauter ces cloisons pour obtenir le vide. Cette technique évite au bois de se fendre sur toute la longueur du tronc. À 18 heures, la future pirogue est déjà creusée sur plus $50 \mathrm{~cm}$ de long et $25 \mathrm{~cm}$ de profondeur. Tambemali a remarqué qu'il y avait une fente (maol mi) dans la partie inférieure de la proue, mais il m'explique que cela n'a pas d'importance, puisque cette partie sera retirée afin de donner le galbe. Il s'attelle ensuite à la fabrication des bordages avant, wanduan kula (litt. "flèche-creuser " ${ }^{17}$ pendant que de nombreux enfants l'observent et ne cessent de lui poser des questions auxquelles il répond avec beaucoup de précision. A 19 heures, à la nuit tombante, Tambemali arrête son travail, et le tronc a déjà pris, en partie, la forme d'une pirogue. 


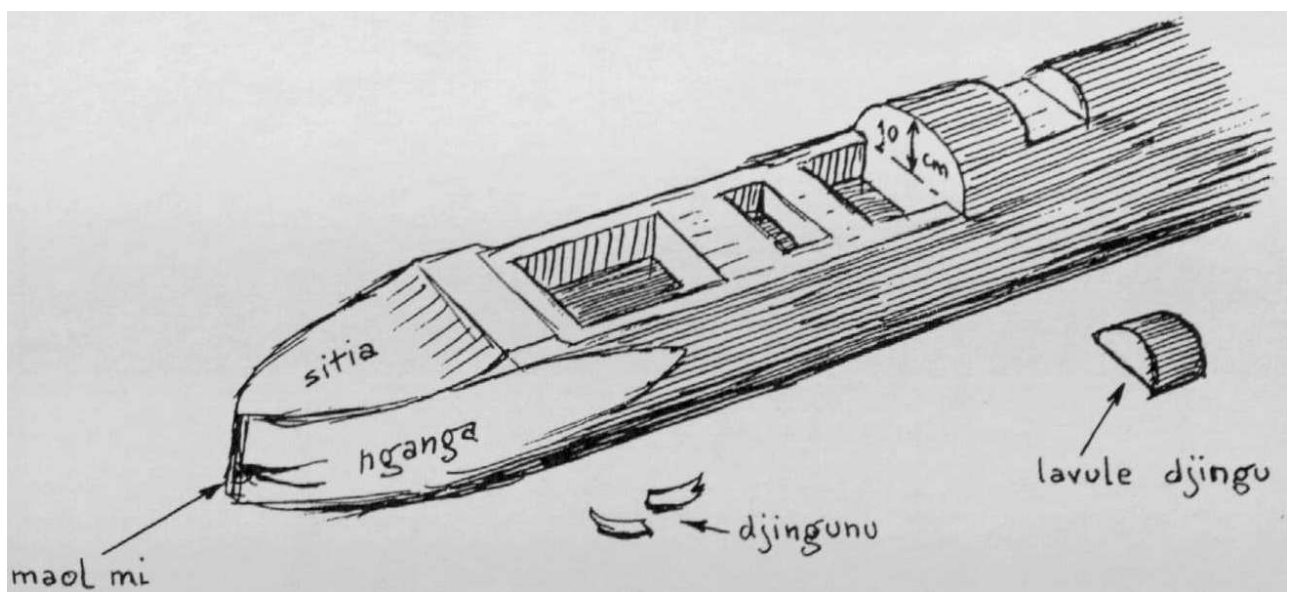

Figure 3. Technique pour creuser un tronc destiné à 1 fabrication d'une pirogue

Le lendemain matin, vers 7 heures 30, Tambemali se remet à l'ouvrage et à 10 heures, la moitié de la pirogue est en forme. Il fait sauter la partie fendue (maol mi) et précise la sculpture des accroches (mutmut); il travaille ainsi toute la matinée à façonner l'intérieur de la poupe en donnant des coups de hache sur les côtés et des coups d'herminettes sur le fond.

À 13 heures 30, il fait une pause pour le repas et la sieste ; il recouvre la future pirogue avec des spathes (mbai) de palmier afin que l'ardeur du soleil ne fasse pas éclater le bois fraîchement découpé. Lorsque Tambemali reprend la fabrication, vers 15 heures, il entreprend de sectionner l'autre extrémité (tambu mi) du tronc, qu'il n'a pas encore traitée, et donne de la sorte sa longueur définitive à la future pirogue. Son plus jeune fils vient le relayer pour la découpe. Lorsque l'ensemble du tronc se trouve ainsi grossièrement creusé, le travail est dit yalgra kula aïmbop (litt. « le ventre est fini d'être creusé »). À 16 heures, les bordages sont déjà bien dégrossis ; Tambemali et son fils arrêtent vers 16 heures 30 (photos 3 et 4).

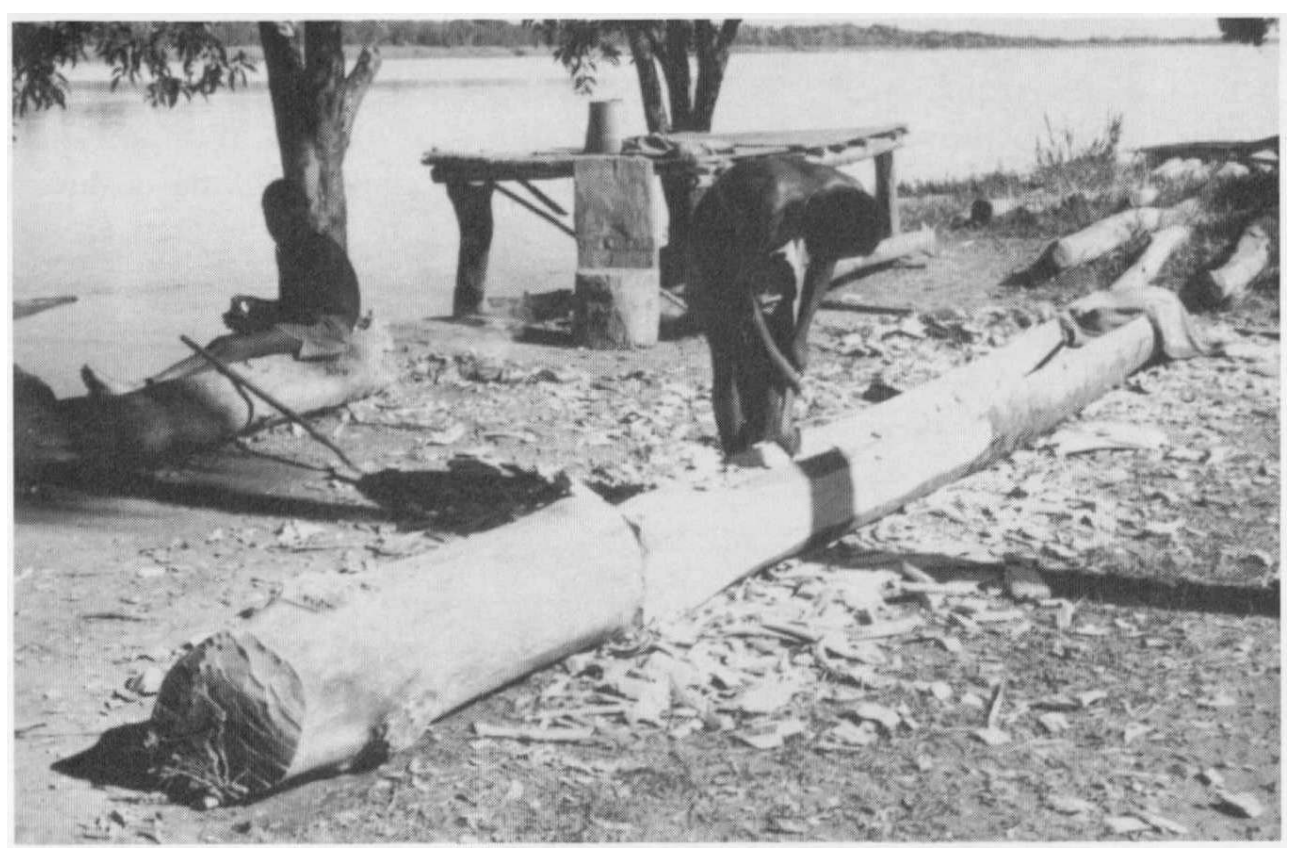




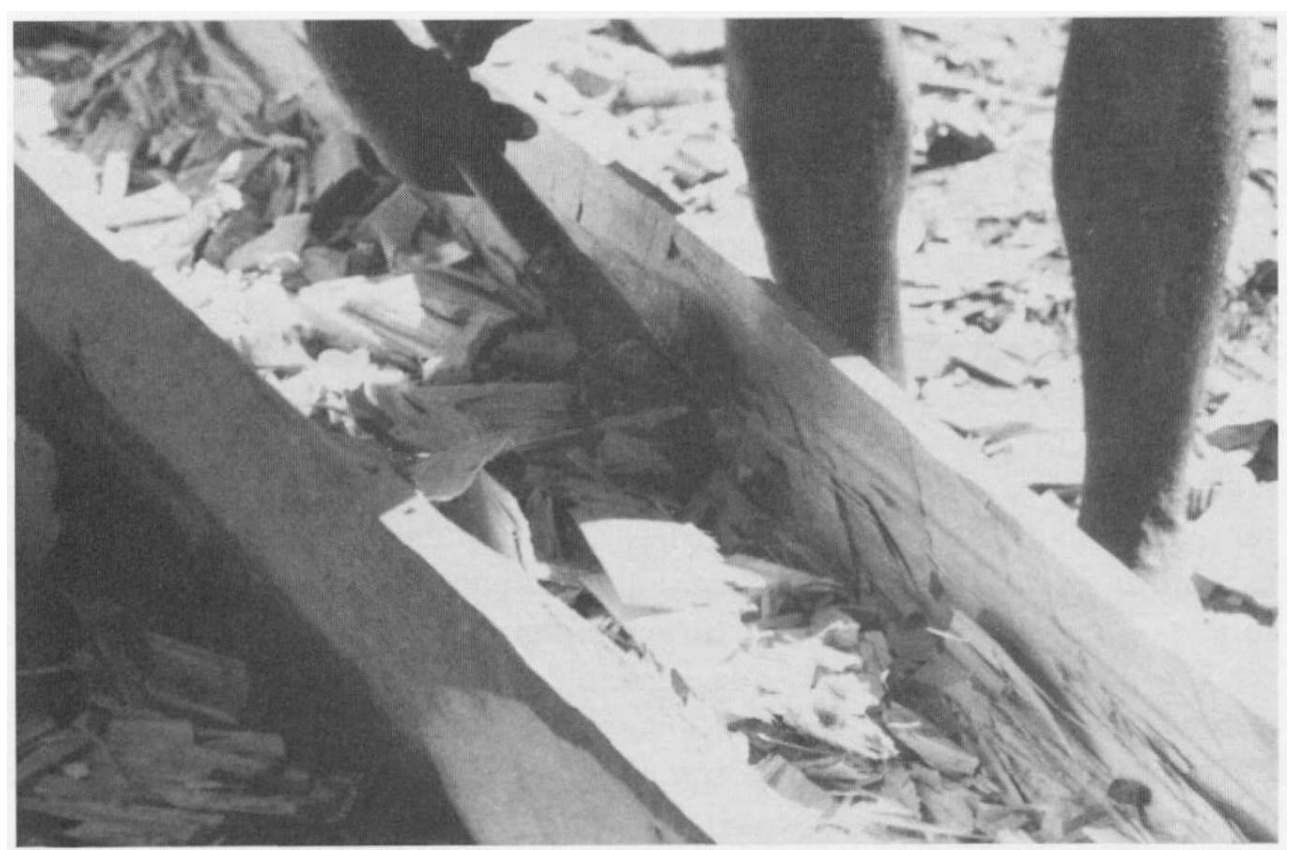

Photos 3 et 4. Taille de l'ébauche d'une pirogue dans un tronc d'arbre de wane (Octomeles sumatrana)

Quelques jours après, sont affinées les parties internes et externes des deux bordages (wamala). Tambemali soigne particulièrement les plats-bords. Les angles entre le fond et les flancs sont appelés manglewala et s'avèrent difficiles à réaliser, car le sculpteur doit constamment contrôler la puissance de ses coups de hache ou d'herminette. Durant cette opération, des barrots d'écartement peuvent être placés de part et d'autre des bordages, à l'intérieur de la pirogue, afin d'en assurer la rigidité. La pirogue est alors retournée de manière à travailler son dos (buni wala) ; c'est une tâche encore plus délicate que la fabrication des bordages et qui requiert une grande expérience : il ne faut pas trop aplanit le bois, au risque de percer son épaisseur, laquelle peut par endroits se restreindre à environ un centimètre. Si c'était le cas, la pirogue deviendrait inutilisable. La résonance de la matière permet d'évaluer son épaisseur.

Les jours suivants, Tambemali sculpte la tête de crocodile de la proue à l'aide d'une petite herminette à lame fine et de ciseaux à bois. Il lui reste à passer toute la surface de la pirogue à la flamme d'un flambeau de feuilles sèches de cocotiers pour durcir le bois. Parfois le bois de la pirogue est entièrement enduit de terre ocre-rouge, koap $\mathrm{mi}^{18}$, pour le rendre plus résistant (Obrist $1984: 1,4,7$ ).

\section{La sculpture de proue}

La pirogue est une représentation du corps d'un crocodile, et par extension, du corps humain. Ce type de représentation se retrouve chez d'autres peuples, comme les Inuit, chez lesquels les noms des divers éléments du kayak évoque également un corps humain ou animal (Therrien 1987 : 49-55). La proue de la majorité des pirogues du Moyen-Sépik est agrémentée d'une figure dont le style varie selon les villages (photo 5). La tête de crocodile est sans conteste le motif le plus fréquent, en particulier sur les petites ou moyennes pirogues; il est généralement appelé mak ndama wala (litt. " petit nez de la pirogue »). Les dimensions de ce motif sont variables: de 35 à $100 \mathrm{~cm}$ de longueur sur 15 à $30 \mathrm{~cm}$ de largeur (Obrist 1984 ; Kelm 1966-68). Les grandes pirogues 
de commerce (autrefois de guerre) présentent à leur proue l'emblème du clan qui les possède ; appelé nema ndama wala (litt. "grand nez de la pirogue »), ce peut être un crocodile, mais aussi un être mythique, comme un poisson, une femme ancêtre (photo 6), ou Gusui, le rat-porc de la pirogue Yamendemba du clan Wanguande.

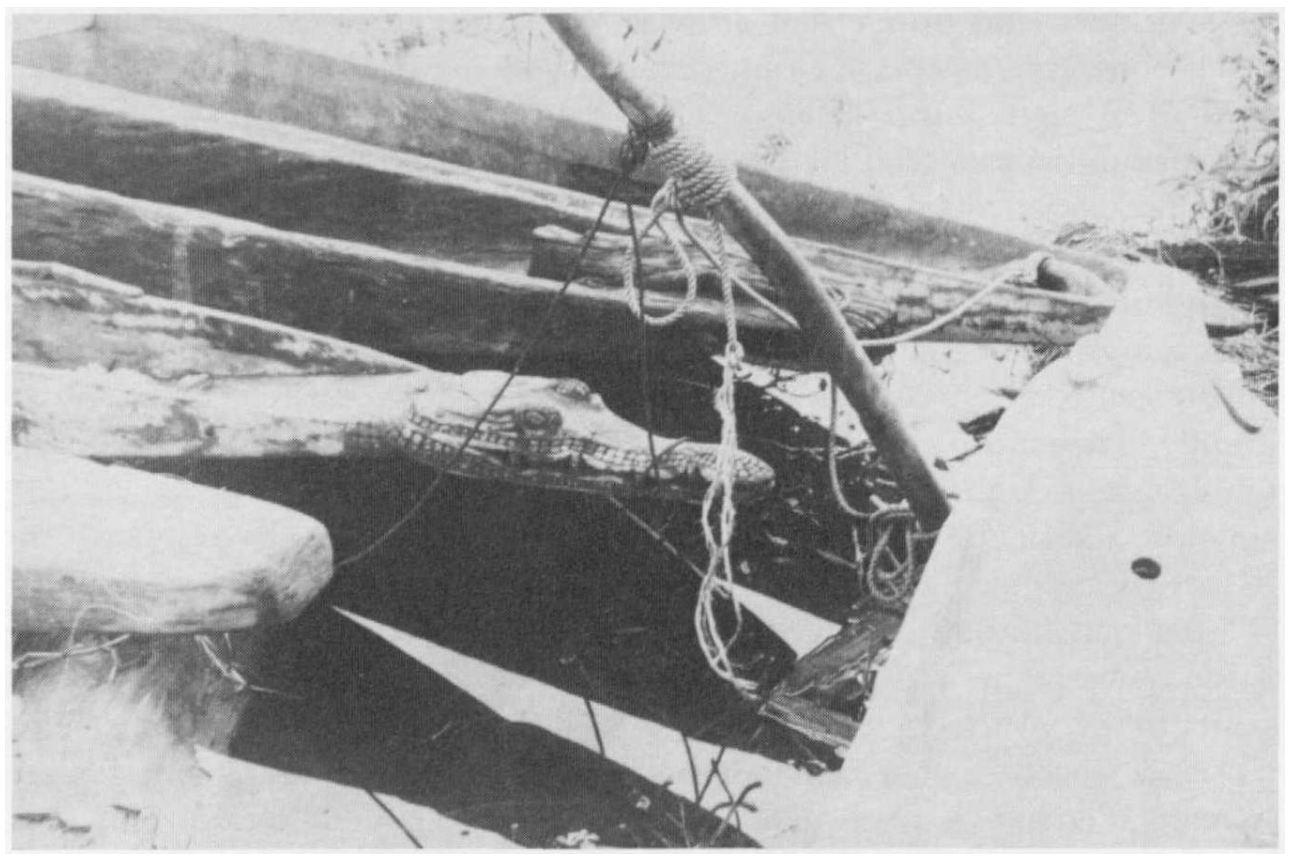

Photo 5. Sculpture de proue d'une pirogue de femme en forme de tête de crocodile

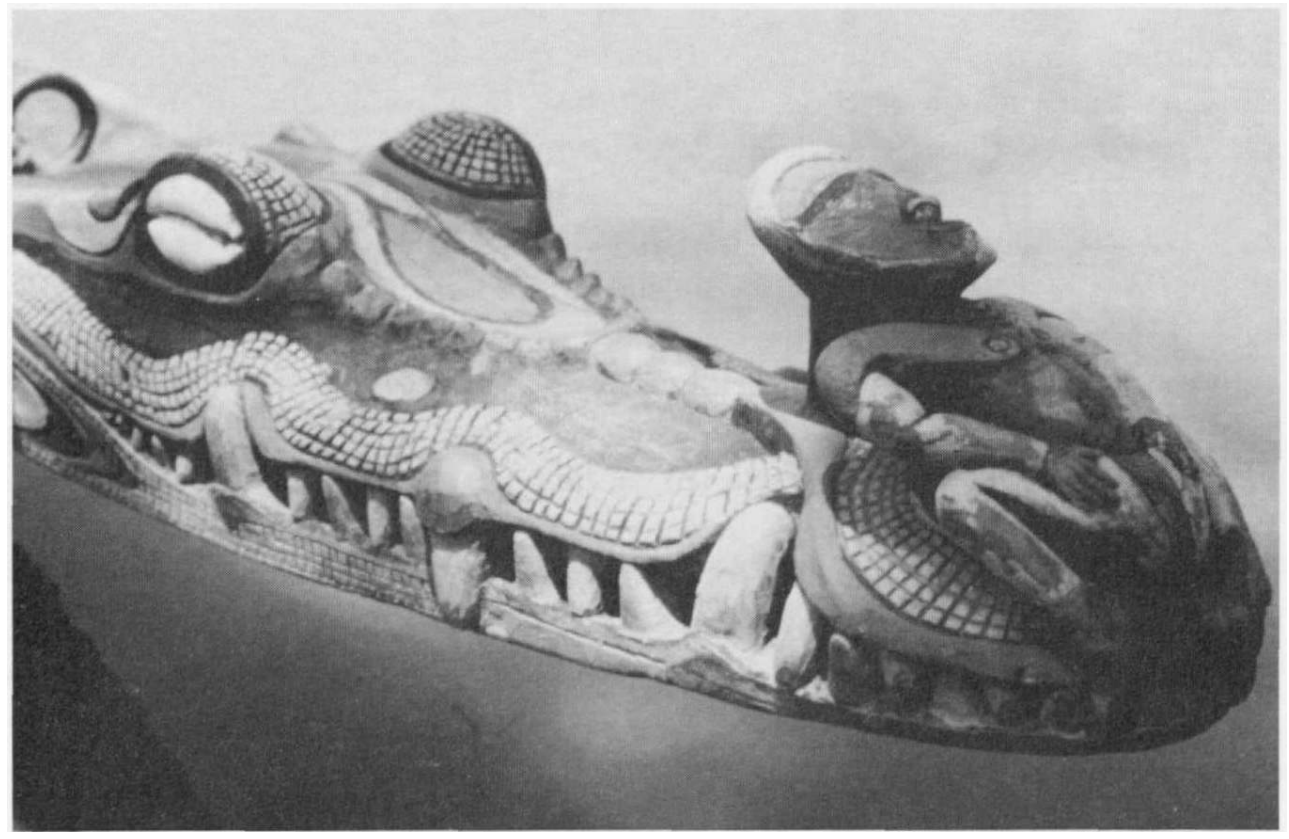

Photo 6. Représentation de Wasandawa à l'extrémité de la tête d'un crocodile, sculptée à la proue d'une pirogue

Lorsqu'un homme n'est pas assez habile pour façonner cette proue, il s'adresse à un bon sculpteur qu'il rétribue avec de la nourriture. Le façonnage de la proue, ndama wala (litt. " nez de la pirogue ») ou wal prouproup (litt. " crocodile-respirer ») et la mise en place de ses yeux (mini wal) en coquillage (sanya) marquent l'achèvement de la construction d'une pirogue. Les Iatmul considèrent qu'une femme ne peut se servir de 
sa pirogue neuve pour aller pêcher, que lorsque les yeux ont été posés. Dans le cas contraire, il lui serait impossible de rapporter du poisson. Jadis, les grandes pirogues comportaient souvent des bordages extérieurs décorés de frises gravées à l'aide d'un instrument tranchant constitué d'une dent de porc. Depuis quelques années, les figures de proue sont fréquemment peintes avec des couleurs chimiques vives provenant de la ville.

\section{Le baptême de la pirogue (wala kwangu)}

Lorsque la pirogue est achevée, elle peut être inaugurée de diverses façons, selon qu'il s'agit d'une petite pirogue de femme (kunguwala) où d'une grande pirogue d'homme (nemawala ou yampuenguewala) et suivant le statut social de l'exécutant. Dans le cas d'une petite pirogue, l'oncle maternel (wau) de l'exécutant vient en dansant glisser ses fesses sur la jambe de ce dernier afin de l'honorer (Bateson 1971). Puis sa mère se rend au marché pour acheter des noix d'arec, du bétel et de la fécule de sagou. À son retour, elle dépose ces divers produits sur la tête de la nouvelle pirogue. Les oncles maternels viennent alors prendre ces cadeaux pour se les partager et les consommer. Ensuite, le fabriquant de la pirogue doit remettre une somme d'argent à son wau qui l'a ainsi honoré. Au village de Palimbeï, la première sortie d'une nouvelle pirogue doit être consacrée à un voyage pour se rendre sur un marché sawos où les femmes échangent, deux fois par semaine, leur surplus de poisson contre de la fécule de palmier sagoutier (Weiss, cité par Obrist 1984 : 4.1.1). Dans le cas d'une grande pirogue, celle-ci doit être décorée de divers emblèmes claniques : feuillages, plumes et coquillages ; puis elle est traînée sur la place cérémonielle où les oncles maternels (wau) du clan propriétaire viennent la consacrer par un naven. Un nom ancestral, en relation avec les autres noms de pirogues du clan, lui est attribué ${ }^{19}$. À cette occasion, les hommes du clan constructeur célèbrent par des chants (wal sagi, litt. " crocodile-chant ») leurs ancêtres communs, au son des longues flûtes évoquant le mythe de la mère originelle et au rythme des batteries de tambours à fente évoquant les ancêtres paternels. Les neveux utétins (lawaniangu), le corps enduit de chaux, viennent apporter des cadeaux qu'ils déposent dans la nouvelle pirogue. Dans certains cas, des hommes peuvent se travestir en esprits féminins d'ancêtres (kaik nyan) en relation avec les flûtes. Ils sont alors superbement parés, comme une jeune mariée, de nombreux bijoux en coquillages, de plumes blanches de poulet et de fruits oranges, (mbuandi, Rejoua aurantiaca), aux couleurs et aux formes variées ${ }^{20}$.

Autrefois, le baptême d'une pirogue de guerre impliquait des rituels très particuliers destinés à assurer la réussite des futures expéditions de " chasse aux têtes ». Un homme était tué et décapité, puis sa tête était déposée à l'intérieur de la nouvelle pirogue à l'avant de laquelle on plaçait une sorte de bouclier de rotin et de spathes, décoré d'une face humaine en bois sculpté (nambu savi). Lorsque la pirogue rentrait ainsi au village, une très grande fête avait lieu avec des danses. Les sœurs et les mères des hommes présents dans la pirogue venaient les frapper avec des faisceaux de feuilles de palmier. Tous les villageois étaient dans la joie. La pirogue étaient amenée sur la place, au milieu du village et la tête de l'homme décapité était déposée en hauteur dans la maison cérémonielle. Le soir, les grands tambours à fente résonnaient dans l'édifice. Les oncles maternels (wau) des hommes ayant participé à l'expédition inaugurale tuaient des cochons et des poulets pour les offrir à leurs neveux (lawa). 


\section{Entretien de la pirogue}

31 fonds vaseux. Les chocs répétés du ressac, les frottements contre les berges et les bordages des autres pirogues arrivent, à la longue, à fendre les plats-bords. Les réparations des petites voies d'eau (wala manguan) s'effectuent avec de l'argile collectée au bord du fleuve. En cas d'avaries plus graves, des plaques de ferraille de récupération (boîtes de conserve en fer blanc) sont fixées par des clous sur la surface défectueuse. L'eau ayant pénétré à l'intérieur de la coque était jadis évacuée avec une spathe (mbai) de palmier. Des fibres de troncs de bananier (mintikmintik) étaient ensuite employées en guise d'éponge. Il semble que les Iatmul ne connaissaient pas les écopes spéciales en bois sculpté, constituées d'une sorte de pelle à manche interne ${ }^{21}$. Ils utilisent actuellement de la filasse et des récipients en plastique de récupération. Régulièrement, le dos d'une pirogue doit être débarrassé des algues vertes (wala kui apma) qui s'y développent en abondance et forment une sorte de gelée gluante. Pour ce faire, la pirogue est sortie de l'eau, renversée sur la berge et grattée à l'aide d'une valve de coquille de moule d'eau douce (wuli). Une pirogue employée régulièrement et bien entretenue ne dure guère plus de cinq ans.

Les éléments des pirogues hors d'usage peuvent être recyclés. Les cercueils sont souvent fabriqués ainsi ; et c'est parfois dans une pirogue que le défunt, dans sa bière, est conduit à sa dernière demeure (Coiffier 1994 : 1330). Les plats-bords brisés peuvent servir de ponts pour accéder aux maisons ou aux toilettes lors de la montée des eaux du fleuve. Les proues sculptées sont sectionnées pour être vendues aux touristes de passage.

\section{Les pagaies}

La propulsion des pirogues est souvent encore assurée manuellement à l'aide de pagaies (ngusa)22 adaptées à leurs utilisateurs. Elles sont façonnées dans des bois très durs, comme le miamba ou le kwarap ${ }^{23}$, qui résistent bien à l'humidité et surtout, dont les fibres présentent une bonne résistance mécanique aux efforts importants auxquels elles seront soumises. Fabriquer une pagaie requiert beaucoup d'expérience. Le bois doit être parfaitement poncé sur toute la surface pour faciliter le glissement dans l'eau d'une part, et pour ne pas blesser les mains du pagayeur d'autre part.

\begin{tabular}{|l|l|}
\hline maknyangu ngusa & pagaie d'enfant à manche court \\
\hline ngal ngusa & pagaie d'homme en forme de queue de poisson \\
\hline yambu ngusa & pagaie d'homme de forme lancéolée \\
\hline tagwa ngusa & pagaie de femme \\
\hline kamblu ngusa & pagaie de chef de pirogue de guerre \\
\hline
\end{tabular}

Divers types de pagaies 


\section{Les représentations de la pirogue}

34 La pirogue n'a pas une fonction exclusivement matérielle: son usage rituel n'est pas rare. Le vocabulaire décrivant les diverses parties d'une pirogue indique, rappelons-le, une identification avec le corps d'un crocodile ou d'un homme. On distingue le dos (buni waala) du ventre (yale waala) dans lequel les occupants s'installent. Les quatre accroches (mutmut) représentent les quatre pattes; elles permettent d'y passer les cordages d'amarrage. Traditionnellement, chaque être humain s'identifie à un crocodile ancestral, qui représente tout à la fois son ego et son lignage paternel. Les occupants d'une pirogue sont considérés comme les enfants du crocodile ancestral. La pirogue est donc perçue comme un crocodile. Si une petite pirogue est identifiée à une personne, une grande pirogue est en revanche associée à un lignage. Les styles des figures de proue varient, nous l'avons vu, selon les villages. Le motif de la tête de crocodile (waal prouproup) est sans conteste la représentation la plus fréquente. Les grandes pirogues présentent souvent des motifs plus complexes, qui évoquent des personnages et des animaux peuplant la mythologie locale. Ces emblèmes permettent d'identifier immédiatement le clan et le village propriétaire de la pirogue, mais ils ont également une fonction de protection. Les termes ndamawala et kengwiwala, désignant la proue et la poupe, sont également employés pour nommer les deux moitiés d'un clan en voie de fission. Répétons que les ndamawala, associés à la tête de l'arbre ancestral, représentent les lignages paternels, alors que les kengwiwala, associés à la base de l'arbre, représentent les lignages maternels.

Concluons sur quelques changements récents. Les moteurs hors-bord introduits dans la région au cours des années soixante-dix ont tout naturellement trouvé leur place à l'arrière de la pirogue, mais leur fixation a posé un problème d'adaptation que les hommes du fleuve ont rapidement résolu. La poupe a dû être modifiée par l'adjonction d'une solide plaque de bois verticale (yangue mi), placée perpendiculairement aux deux bordages parallèles. Cette plaque est le plus souvent réalisée en bois de Vitex cofassus, propre à résister aux vibrations et aux diverses manipulations du moteur lors du nettoyage de l'hélice (Coiffier 1995a : 69-70).

La pirogue est encore aujourd'hui le moyen de transport indispensable à tous les villageois riverains du fleuve Sépik. Les nouvelles embarcations en métal ou en fibres de verre ne sont aucunement adaptées aux usages locaux. Elles sont trop larges pour pouvoir se frayer un passage à travers les hautes herbes des berges et les multiples canaux où vont pêcher les femmes. La fabrication des pirogues monoxyles reste donc une activité vitale pour ces populations de pêcheurs. Celles-ci peuvent se procurer la matière première sur place et recycler ensuite les pirogues usagées. Les débris de pirogues retournent à la terre ou dans la vase du fleuve pour constituer un terreau fertile qui favorisera la croissance de nouveaux arbres. Ainsi se perpétuent de longues lignées de pirogues qui se confondent avec les lignages familiaux, les unes comme les autres issues de l'ancêtre crocodile primordial. 
Annexes : Essences d'arbres utilisées pour la fabrication des pirogues. ${ }^{24} 25$

\begin{tabular}{|l|l|l|}
\hline Nom scientifique & Nom vernaculaire & Consistance \\
\hline Vitex cofassus & miamba & très dur et dense \\
\hline Intsia bijunga 23 & kwarap & très dur et dense \\
\hline Dysoxylum sp. & tshambandaoun & dur \\
\hline Serianthes sp. & yao & dur \\
\hline Pterocarpus indicus & didjanguan & dur \\
\hline Terminalia sepikana & kwi & moyennement dur \\
\hline Terminalia catappa & karaba & moyennement dur \\
\hline Terminalia sp. & kusik & moyennement dur \\
\hline Octomeles sumatrana & wane & dur \\
\hline Adenanthera novo.guineensis & lukuit & dur \\
\hline Alstonia scholaris & kambu & tendre \\
\hline Artocarpus altilis & kam & tendre \\
\hline Cinnamomum sp. & nguambi & dur \\
\hline Cordia sp. & djambi & dur \\
\hline Dracontomelon sp. & tschuiangue & - \\
\hline Ficus sp. & kuambi & - \\
\hline Homalium foetidum & mengue & très dense \\
\hline Neonauclea sp. 24 & ngupma & très dense \\
\hline Pangium edule & ngangrap & - \\
\hline Poinciana delnis & malmal (pid.) & moyennement dur \\
\hline Syzygium sp. & kuikuin & dur \\
\hline Syzygium cf. malaccense & kukuan & dur \\
\hline & & \\
\hline
\end{tabular}

37 Nota: les dix premiers noms cités dans la liste correspondent aux meilleurs bois classés par ordre d'importance par un informateur du village de Yentchen. Selon Foreman (1972 : 1131), les espèces les plus couramment utilisées en Papouasie Nouvelle-Guinée pour la fabrication des pirogues sont Elmerrillia papuana, Octomeles sumatrana et Toona sureni.

\section{BIBLIOGRAPHIE}

Bateson, Gregory, 1971, La Cérémonie du naven. Paris : Éditions de Minuit (1ère édition 1936).

Best, Elston, 1925, The Maori Canoe. Wellington : Dominion Museum (Bulletin n7).

Coiffier, Christian, 1994, Tshimbe kuvu, kwiya kuvu. L'écorce et la moelle du rotin. Conception iatmul de l'univers. Thèse de Doctorat en anthropologie sociale et ethnologie. Paris : EHESS. - 1995a, " "Le crocodile à moteur", les pirogues et leur motorisation chez les Iatmul du fleuve Sépik (Papouasie Nouvelle-Guinée) ", Anthropologie Maritime 5 : 63-78 (Les hommes et les bateaux, Usages, appropriation et représentations).

- 1995b, « Les îles flottantes du fleuve Sépik » (note concernant l'article de Henri Lavondès : Jules Verne, les Polynésiens et le motif de l'île mouvante : Miscellanées), Journal de la Société des Océanistes 100-101:238-240.

- 2000, « La femme-crocodile et son frère », pp. 195-213, in J. Cauquelin (éd.), L'énigme conjugale. Femmes et mariage en Asie. Clermond-Ferrand : Presses Universitaires Blaise Pascal. 
Coiffier, Christian et Antonio Guerreiro, 1999, « La chasse aux têtes : une dette de vie », pp. 30-45, in Catalogue de l'exposition «La mort n'en saura rien ». Reliques d'Europe et d'Océanie. Paris : Réunion des Musées Nationaux.

Foreman, D. B., 1972, « Timber, commercial species », pp. 1124-1134, in P. Ryan (ed.), Encyclopaedia of Papua New Guinea. Melbourne : Melbourne University Press/University of Papua and New Guinea.

Fuerst, René, 1988, Navigateurs des mers du sud. Genève : Musée d'Ethnographie (Catalogue d'exposition).

Haberland, Eike, 1968, The Caves of Karawari. New-York : D'Arcy Galleries. Kelm, Heinz - 1966-68, Kunst vom Sepik, Vol. I, Neue Folge 10, Abteilung Südsee V, Vol. II, Neue Folge 11, Abteilung Südsee VI \& Vol. III, Neue Folge 15, Abteilung Südsee VII. Berlin : Museum für Völkerkunde.

Lupu, François, 1980, « Toponymies Tin-Dama », pp. 182-187, in Cartes et figures de la terre. Paris : CCI, Centre Georges Pompidou.

Mordant, D., 1993, « Une pirogue carolingienne à Noyen sur-Seine », Archéologia 287 : 4. Pétard, Paul

- 1986, Plantes utiles de Polynésie. Raaut tahiti. Papeete (Tahiti) : Éditions Haere Po No Tahiti.

Noël, Michel et Aimé Bocquet

- 1987, Les Hommes et le bois. Histoire et technologie du bois de la préhistoire à nos jours. Paris : Hachette (« La mémoire du Temps »).

Obrist, Brigit

- 1984, Iatmul. Objektkatalog, Sepik. Dokumentation. Basel : Museum für Völkerkunde (Multigr.).

Simutoga, Pierre Chanel

- 1992, Technologie traditionnelle à Wallis. Essai de sauvegarde de la mémoire collective des charpentiers wallisiens du district de Hihifo. Paris : Musée de l'Homme (Société des Océanistes, n44).

Stanek, Milan, 1977, « Vara kwangu. Le baptême de la pirogue », pp. 52-57, in E. von Herausgegeben Lichtenhahn et F. Borei (eds), Musique et Sociétés. Neuchâtel : Musée d'ethnographie.

Therrien, Michèle, 1987, Le Corps des Inuit (Québec arctique). Paris : Éditions SELAF.

\section{NOTES}

2. Le terme Iatmul a été employé par Bateson pour désigner un groupe linguistique qui se reconnaît comme une entité distincte de ses voisins sous le nom de Iatmuliambonaï. Les Iatmul parlent une langue non austronésienne. Du début du siècle jusqu'à l'indépendance de l'état Papou, en 1975, cette société fut confrontée à la colonisation, allemande puis australienne.

3. Autrefois, à l'époque de la "chasse aux têtes", les guerriers utilisaient de grandes pirogues pour organiser des raids sur les villages ennemis et pour aller tendre des embuscades en se cachant dans les hautes herbes des berges du fleuve (Coiffier 1995a : 68).

4. Un informateur m'a raconté que ses ancêtres avaient utilisé, jadis, des pirogues à balanciers (Coiffier 1994 :126). Il est vrai que parfois, les populations des affluents du fleuve Sépik, comme le Keram, naviguent encore sur des pirogues à balanciers. 
5. Fabriquer une pirogue se dit wala kapma vialeko en langue iatmul, soit littéralement " piroguefrapper». Dans un article (1995a), j'avais transcrit le mot pirogue par le terme waala. Après vérification sur le terrain, il faut écrire wala.

6. Divers types de flûte peuvent être utilisés pour ce rituel, et particulièrement celles évoquant les aboiements de chien. Les longues flûtes sont également associées aux esprits wundjumbu (Coiffier $1994: 870$ ).

7. Ainsi, les flûtes sont perçues comme la voix des ancêtres maternels alors que les tambours à fente évoquent les ancêtres paternels (Coiffier $2000: 198$ ).

8. Chez les Tin dama (population voisine des Iatmul établie sur les rives du fleuve Keram), «Une fois à terre, l'arbre est ébranché et tout ce qui se trouve près de la souche est soigneusement nettoyé et recouvert de peinture rouge » (Lupu 1980 : 185-186).

9. Autrefois, les jeunes Iatmul fabriquaient leur première pirogue à l'âge de 11, 12 ou 13 ans, lorsqu'ils entraient dans le mbolé, maison réservée aux adolescents. Aujourd'hui, avec le développement de la scolarisation, ce n'est que vers de 15 ou 17 ans qu'ils font l'apprentissage de cette pratique; mais la réalisation d'une pirogue constitue toujours un acte important dans la socialisation d'un jeune garçon. Ainsi, l'oncle maternel de l'enfant, son wau, se doit de lui témoigner sa satisfaction en organisant un rituel naven (Bateson 1971). Un homme est toujours très fier d'annoncer le nombre de pirogues qu'il a sculptées au cours de sa vie.

10. Ainsi la route de wane (Octomeles sumatrana), ancêtre du clan Kwanguwala-Suaru, vient du village Yamok par l'affluent Wanewulden.

11. Noël et Bocquet (1987:53) donnent un volume de 1,5 à plus de $5 \mathrm{~m}^{3}$ pour les pirogues du néolithique européen.

12. Pour un exemple de fabrication d'une pirogue à Wallis, $c f$. Simutoga (1992 : 121-163).

13. Raphaël Tambemali Tonakuan et son épouse Mblimbo vivaient lors de mon arrivée au village de Palimbeï, en 1987, dans une maison voisine de celle de mes hôtes. Ils ont 42 petits-enfants de leurs 17 enfants. Tambemali est un homme d'une soixantaine d'années, qui a passé une partie de sa vie dans une mission catholique où il exerça la fonction de cathéchiste. C'est un homme affable et pondéré dans ses jugements. Malgré des lacunes dans le savoir ésotérique de son clan, dû à son éloignement du village durant de nombreuses années, c'est un homme qui possède de grandes connaissances sur sa propre culture (Coiffier 1994 : 212). Le récit de cette fabrication, qui eut lieu au début de l'année 1988, est extrait de ma thèse de doctorat (Coiffier 1994 : 1124-1127).

14. Ce qui correspond au cas général de l'utilisation de troncs pour la fabrication des pirogues monoxyles : la poupe à la souche et la proue du côté du houppier. La découverte récente d'une pirogue de l'époque carolingienne à Noyen-sur-Seine (France), qui fut taillée de cette façon, vient confirmer ce mode d'utilisation des troncs pour l'Europe médiévale (Mordant 1993 : 4).

15. Les occupants des pirogues, particulièrement les femmes, s'assoient sur la partie poupe de la pirogue, qui s'enfonce légèrement dans l'eau. C'est donc cette partie qui frotte avant les autres les fonds limoneux lorsque la pirogue aborde une berge.

16. Selon un mythe du peuple Ymar (installé sur les rives de la rivière Karawari), les copeaux provenant de la taille d'un grand tambour à fente se transformèrent en créatures démoniaques, appelées yip'wons, et associées à la chasse aux têtes et à la guerre (Haberland 1968 : XIX). Selon Best (1925: 312), lorsque les Maori fabriquaient une pirogue de guerre, le premier copeau arraché lors de l'abattage de l'arbre était immédiatement emporté au loin dans la forêt par un homme qui le brûlait dans un feu, obtenu par friction, en chantant des paroles rituelles. Ce rite était destiné à Tane, Dieu des artisans. Ultérieurement, les autres copeaux étaient également brûlés.

17. La réunion des deux bordages de la pirogue évoque en effet la forme d'une pointe de flèche.

18. Il est probable qu'il s'agisse de terre mélangée avec de la sève de l'arbre kuoop mi (Sloanea sp.).

19. La généalogie de ces pirogues se trouve ainsi en parallèle avec celle des humains qui les ont fabriquées et utilisées. Ainsi, la lignée de toutes les pirogues du clan Ndamara-Suaru, auquel 
appartient mon informateur, Temben, du village de Palimbeï, s'appelle kengwiwala yalanguenemba yalenga.

20. La plupart des informations sur le baptême d'une grande pirogue sont extraites d'un article de Stanek (1977 : 52-57) ; cf. également Bateson (1971).

21. Les écopes existaient cependant dans le Bas-Sépik et dans la région de la rivière Karawari (Kelm 1966/68, I : 416, Fuerst $1988: 175-179)$. Ce type d'objet était répandu dans une vaste zone de l'Océanie.

22. Le terme ngusa évoque l'idée de fendre l'eau, soit littéralement : ngu-sa = eau-séparer.

23. Mais d'autres bois durs peuvent être utilisés: djidjangua, tshambandaun, yao, maapu (Artocarpus sepicanus ?), mbangla kase (Cordia sp. ?).

24. À Fidji, les coques des grandes pirogues de mer était entièrement réalisées en Intsia bijunga (Hélène Guyot, communication personnelle).

25. Le bois de Neonauclea était également utilisé à Tahiti par les constructeurs de pirogues (Pétard $1986: 276)$

\section{RÉSUMÉS}

La pirogue monoxyle constitue, pour les communautés de pécheurs riveraines du fleuve Sépik, un indispensable moyen de transport, en adéquation avec leur environnement aquatique. La pirogue permet aux femmes de se rendre sur leurs lieux de pêche, elle assure en outre le transport des personnes et des marchandises entre les différents villages. Il existe plusieurs types de pirogue, adaptés à divers usages. Toutes cependant sont fabriquées suivant les mêmes techniques et à partir d'un tronc d'arbre. Afin de mieux comprendre l'importance de cette embarcation, on évoquera ses rapports avec l'univers mythique local, où elle se confond avec l'ancêtre crocodile primordial.

The monoxylon pirogue represents, for the river fishing communities of the Sepik river, the indispensable mean for transport and it is the most well adapted to their aquatic environment. The pirogue makes it possible for the women to go to their fishing area and also insures the transport of people and merchandises between the different villages. Several types of pirogues exist that are adapted to the different uses. However, all of them are constructed following the same technics and are chosen from the trunks of trees that have different lengths. In order to better understand the importance of this boat, we will mention its relations to the local mythical world, where it is intermingled with the primordial Crocodile Ancestor.

La piragua monoxila representa, para las comunidades de pescadores rivereños del río Sepik, el medio de transporte indispensable y el mejor adaptado a su medio ambiente acuático. La piragua permite a las mujeres desplazarse a sus lugares de pesca, garantiza también el transporte de personas y mercancías entre los distintos pueblos. Existen varios tipos de piraguas, adaptables a diversos usos. Sin embargo, todas son construidas según las mismas técnicas y a partir de un tronco de árbol de largo más o menos grande. A fin de comprender mejor la importencia de esta embarcación, se evocarán sus relaciones con el universo mítico local donde se confunde con el Ancestro crocodrilo primordial. 
INDEX

Mots-clés : monoxyle, Iatmul, pirogue, pêche, Papouasie Nouvelle-Guinée, Sépik.

\section{AUTEUR}

\section{CHRISTIAN COIFFIER}

Laboratoire d'ethnologie du Musée de l'Homme/Techniques et culture, 27 rue Paul Bert, 94200 Ivry-sur-Seine 